TRANSACTIONS OF THE

AMERICAN MATHEMATICAL SOCIETY

Volume 350, Number 8, August 1998, Pages 3371-3391

$\mathrm{S} 0002-9947(98) 02017-0$

\title{
WEIGHTED NORM INEQUALITIES FOR INTEGRAL OPERATORS
}

\author{
IGOR E. VERBITSKY AND RICHARD L. WHEEDEN
}

\begin{abstract}
We consider a large class of positive integral operators acting on functions which are defined on a space of homogeneous type with a group structure. We show that any such operator has a discrete (dyadic) version which is always essentially equivalent in norm to the original operator. As an application, we study conditions of "testing type," like those initially introduced by E. Sawyer in relation to the Hardy-Littlewood maximal function, which determine when a positive integral operator satisfies two-weight weak-type or strong-type $\left(L^{p}, L^{q}\right)$ estimates. We show that in such a space it is possible to characterize these estimates by testing them only over "cubes". We also study some pointwise conditions which are sufficient for strong-type estimates and have applications to solvability of certain nonlinear equations.
\end{abstract}

\section{INTRODUCTION}

Our main purpose in this paper is to continue studying conditions which determine when a positive integral operator satisfies two-weight weak-type or strong-type $\left(L^{p}, L^{q}\right)$ estimates. We consider integral operators of the form

$$
T(f d \sigma)(x)=\int_{X} K(x, y) f(y) d \sigma(y), \quad x \in X,
$$

where $X$ is a space of homogeneous type in the sense of [CW] which is also equipped with a group structure (a precise definition is given below), $\sigma$ is a nonnegative Borel measure, and $K(x, y) \geq 0$. If $K$ satisfies some further restrictions, we will define a dyadic version of $T$ which is pointwise smaller than $T$ but still essentially equivalent to $T$ in norm. We will use the dyadic version of $T$ to study both the weak-type estimate

$$
\rho|\{x \in X: T(f d \sigma)(x)>\rho\}|_{\omega}^{1 / q} \leq c\|f\|_{L_{\sigma}^{p}}, \quad \rho>0,
$$

and the strong-type estimate

$$
\|T(f d \sigma)\|_{L_{\omega}^{q}} \leq c\|f\|_{L_{\sigma}^{p}}
$$

in case $1<p \leq q<\infty$. Here $\omega$ and $\sigma$ are nonnegative Borel measures on $X,|E|_{\omega}$ denotes the $\omega$-measure of a set $E$, and

$$
\|f\|_{L_{\sigma}^{p}}=\left(\int_{X}|f(x)|^{p} d \sigma(x)\right)^{1 / p}, \quad 1 \leq p<\infty .
$$

Received by the editors March 28, 1996 and, in revised form, October 1, 1996.

1991 Mathematics Subject Classification. Primary 42B20, 42B25.

The first author was partially supported by NSF Grant DMS94-01493 and the second by NSF Grant DMS95-00799.

(C)1998 American Mathematical Society 
We also treat the case $p=1$ and $1<q<\infty$ for the weak-type estimate. In order to avoid obvious complications which occur when $\sigma$ and $\omega$ have joint atoms, a standing assumption throughout the paper will be that $\sigma$ vanishes on sets which consist of a single point. We will use this assumption wherever the dyadic version of $T$ is involved.

We will first study some further conditions of "testing type", as defined below, on the measures $\omega, \sigma$ which completely determine when (1.2) or (1.3) holds. Conditions of this type were initially introduced by E. Sawyer in [S1] in relation to the HardyLittlewood maximal function, and later studied in [S2], [S3] in relation to fractional integrals in case $X$ is Euclidean space, and they have been studied recently in more generality in [SW], [SWZ] and [WZ]. Some conditions of a different type were also studied in [SW] and [SWZ] (see also the references in these papers).

For our results about testing conditions, we are specifically interested in showing that it is possible to characterize (1.2) or (1.3) by testing these estimates only over "cubes", in a sense which we shall make precise. It is proved in [WZ] that it is enough to test over balls in any space of homogeneous type, even if there is no group structure. Although cubes and balls are essentially equivalent from the point of view of containment, it is not obvious that testing with balls and testing with cubes are equivalent characterizations for the norm estimates, since the measures $\omega, \sigma$ are not assumed to satisfy doubling conditions.

We will also study some pointwise conditions like those in $[\mathrm{MV}]$, [VW] and [KV] which are sufficient in order for the strong-type estimate (1.3) to hold in case $p=q$. These conditions imply not only (1.3), but also the inequalities

$$
\|T(f d \sigma)\|_{L_{\omega_{j}}^{p}} \leq c_{j}\|f\|_{L_{\sigma}^{p}}, \quad j=0,1,2, \ldots,
$$

where $\omega_{0}=\omega$ and $d \omega_{j}=\left[T\left(\mathbf{1} d \omega_{j-1}\right)\right]^{p^{\prime}} d \sigma(j=1,2, \ldots)$. Here $1 / p+1 / p^{\prime}=1$ and

$$
T(\mathbf{1} d \omega)(x)=\int_{X} K(x, y) d \omega(y), \quad x \in X .
$$

Inequalities of this type arise in applications of weighted norm inequalities to the problem of the existence of nonnegative solutions for the "superlinear" integral equation

$$
u(x)=\int_{X} K(x, y) u(y)^{q} d \sigma(y)+f(x), \quad x \in X,
$$

where $q>1$ and $f$ is a nonnegative measurable function on $X$. The pointwise conditions are necessary and sufficient for the solvability of the equation above and in some cases are also necessary for (1.3) to hold. (See [VW] and [KV].)

In order to be more precise, we now list some relevant definitions. A homogeneous space $(X, d, \mu)$ in the sense of $[\mathrm{CW}]$ is a set $X$ together with a quasi-metric $d$ and a doubling measure $\mu$. By a quasi-metric, we mean a mapping $d: X \times X \rightarrow[0, \infty)$ which satisfies

$$
\begin{aligned}
& d(x, y)=0 \quad \text { if and only if } \quad x=y, \\
& d(x, y)=d(y, x) \text { for all } x, y \in X, \\
& d(x, y) \leq \kappa(d(x, z)+d(z, y)) \text { for all } x, y, z \in X,
\end{aligned}
$$

where $\kappa \geq 1$ is a constant which is independent of $x, y$, and $z$. By a doubling measure $\mu$ on $X$ we mean a (locally finite) nonnegative measure on the Borel subsets of $X$ so that $|B(x, 2 r)|_{\mu} \leq C_{\mu}|B(x, r)|_{\mu}$ for all $x \in X$ and $r>0$, where $B(x, r)=$ 
$\{y \in X: d(x, y)<r\}$ is the ball centered at $x$ with radius $r$, and $|B(x, r)|_{\mu}$ is the $\mu$-measure of $B(x, r)$. The constant $C_{\mu}$ is called the doubling constant of $\mu$ and is independent of $x$ and $r$. We assume that all balls $B(x, r)$ in $X$ are open. We shall also assume that all annuli $B(x, R) \backslash B(x, r)$ in $X$ are nonempty for $0<r<R$. As usual, if $B=B(x, r)$ and $c>0$, we denote by $c B$ the ball $B(x, c r)$.

It has been proved in [SW] that for $\lambda=8 \kappa^{5}$, and for any (large negative) integer $m$, there are points $\left\{x_{j}^{k}\right\}$ and a family $\mathcal{D}_{m}=\left\{E_{j}^{k}\right\}$ of sets for $k=m, m+1, \ldots$ and $j=1,2, \ldots$ such that

$$
B\left(x_{j}^{k}, \lambda^{k}\right) \subset E_{j}^{k} \subset B\left(x_{j}^{k}, \lambda^{k+1}\right),
$$

$$
\begin{array}{r}
X=\bigcup_{j} E_{j}^{k} \text { and }\left\{E_{j}^{k}\right\} \text { are pairwise disjoint in } j, \\
\text { (iii) If } k<l \text { then either } E_{j}^{k} \cap E_{i}^{l}=\emptyset \text { or } E_{j}^{k} \subset E_{i}^{l} .
\end{array}
$$

We shall say that the family $\mathcal{D}=\bigcup_{m=-\infty}^{\infty} \mathcal{D}_{m}$ is a dyadic cube decomposition of $X$, and call sets in $\mathcal{D}$ dyadic cubes and denote them by $Q$. We note that the cubes in $\mathcal{D}_{m_{1}}$ may have no relation to those in $\mathcal{D}_{m_{2}}$ if $m_{1}$ and $m_{2}$ are different. If $Q=E_{j}^{k} \in \mathcal{D}_{m}$ for some $m$, we say $Q$ is centered at $x_{j}^{k}$, and define the sidelength of $Q$ to be $\ell(Q)=\lambda^{k}$. We also denote by $Q^{*}$ the containing ball $B\left(x_{j}^{k}, \lambda^{k+1}\right)$ of $Q$. A construction of a dyadic grid which includes nestedness even for arbitrarily small cubes is given in $[\mathrm{C}]$, but in the properties listed there, the cubes of a given size may not completely cover $X$, omitting a subset of $\mu$-measure zero, which may not be adequate when we deal with measures that are not absolutely continuous with respect to $\mu$.

We will usually assume that $(X, d, \mu)$ has a group structure with respect to the operation "+", and that the following properties are satisfied for all $x, y, z \in X$ and all balls $B$ in $X$ :

$$
\begin{aligned}
& \text { (1) } d(x+z, y+z)=d(x, y), \\
& \text { (2) }|-B+z|_{\mu}=|B|_{\mu}, \quad \text { where } \quad-B=\{x \in X:-x \in B\} .
\end{aligned}
$$

The group may not be commutative, and we could replace the right invariance in (1.6) by left invariance.

The kernel $K(x, y)$ of $T$ is always assumed to be a nonnegative function which satisfies

$$
\begin{aligned}
& K(x, y) \leq C_{1} K\left(x^{\prime}, y\right) \text { if } \quad d\left(x^{\prime}, y\right) \leq C_{2} d(x, y), \\
& K(x, y) \leq C_{1} K\left(x, y^{\prime}\right) \quad \text { if } \quad d\left(x, y^{\prime}\right) \leq C_{2} d(x, y),
\end{aligned}
$$

where $C_{1}, C_{2}$ are fixed constants larger than 1.

One of our main results involves a new discrete (dyadic) version of the operator $T(f d \sigma)$, which we now discuss. A more thorough discussion is given in the next section. If $B$ is a ball in $X$ with radius $r(B)$, we define (see [SW])

$$
\phi(B)=\sup _{\substack{x, y \in B \\ d(x, y) \geq c r(B)}} K(x, y),
$$

where $c$ is a suitably small positive constant depending only on the quasimetric constant $\kappa$. Let $\mathcal{G}$ denote any fixed dyadic grid of cubes $Q$. Typically, $\mathcal{G}=\mathcal{D}_{m}+z$, 
i.e.,

$$
\mathcal{G}=\left\{E_{j}^{k}+z: k \geq m, j \geq 1\right\}
$$

for fixed $m$ and $z$, where the cubes $E_{j}^{k}$ are as in (1.5). Given $x \in X$ and a cube $Q$ in $\mathcal{G}$ which contains $x$, define $P(Q, x)$ to be the next smaller cube in $\mathcal{G}$ which contains $x$, i.e., if the sidelength of $Q$ is $\lambda^{k}$, then $P(Q, x)$ is the cube in $\mathcal{G}$ which contains $x$ whose sidelength is $\lambda^{k-1}$; if no such cube exists (i.e., if $Q$ is a minimal cube in $\mathcal{G}$ ), define $P(Q, x)$ to be empty. Of course, $P(Q, x)$ also depends on $\mathcal{G}$, but we shall not display this dependence in the notation. The dyadic version of $T(f d \sigma)$ which will play an important role in our results is defined by

$$
t_{\mathcal{G}}(f d \sigma)(x)=\sum_{Q \in \mathcal{G}: x \in Q} \phi_{Q} \int_{Q \backslash P(Q, x)} f(y) d \sigma(y),
$$

for $f \geq 0$, where $\phi_{Q}$ is defined to be $\phi\left(Q^{*}\right), Q^{*}$ being the containing ball of $Q$ defined earlier (see (1.5)). This function is clearly pointwise smaller than the closely related function

$$
T_{\mathcal{G}}(f d \sigma)(x)=\sum_{Q \in \mathcal{G}: x \in Q} \phi_{Q} \int_{Q} f(y) d \sigma(y),
$$

formed by integrating over all of $Q$ rather than over just $Q \backslash P(Q, x)$. We shall see that $t_{\mathcal{G}}(f d \sigma)$ is also pointwise smaller than $T(f d \sigma)$ but still essentially comparable in norm to $T(f d \sigma)$. We will prove the following result, in which we use the standard notation

$$
\|f\|_{L_{\omega}^{q, \infty}}=\sup _{\rho>0} \rho|\{x \in X:|f(x)|>\rho\}|_{\omega}^{1 / q}
$$

in case $1<q<\infty$.

Theorem 1.1. Let $1 \leq q \leq \infty$, and let $\sigma$ and $\omega$ be locally finite Borel measures on a homogeneous space $X$ with a group operation "+" which satisfies (1.6). Let T be defined by (1.1) with a kernel which satisfies (1.7). Then for all $x \in X$ and any dyadic grid $\mathcal{G}$,

$$
t_{\mathcal{G}}(f d \sigma)(x) \leq C T(f d \sigma)(x)
$$

with $C$ depending only on $\kappa$ and the constants in (1.7), but not on $\mathcal{G}, x, f$, or $\sigma$. Moreover,

$$
\|T(f d \sigma)\|_{L_{\omega}^{q}} \leq C \sup _{z \in X, m \in \mathbf{Z}}\left\|t_{\mathcal{D}_{m}+z}(f d \sigma)\right\|_{L_{\omega}^{q}}
$$

with $C$ independent of $f, \sigma$ and $\omega$. Thus the last inequality is an equivalence, with constants of equivalence which are independent of $f, \sigma$ and $\omega$; this equivalence also holds with $\|\cdot\|_{L_{\omega}^{q}}$ replaced by $\|\cdot\|_{L_{\omega}^{q, \infty}}$ if $1<q<\infty$.

The larger function $T_{\mathcal{G}}(f d \sigma)$ was considered in [SW] and [SWZ], and is similarly related to $T(f d \sigma)$ with one drawback: the pointwise estimate $T_{\mathcal{G}}(f d \sigma)(x) \leq$ $c T(f d \sigma)(x)$ is only known to hold with an extra hypothesis on $K$ (see (1.24) of $[\mathrm{SW}])$.

It is worth noting that the discrete operator $t_{\mathcal{G}}$ has a symmetric kernel (see section 2), while this is not necessarily the case for $T$. We shall denote the adjoint of $T$ by $T^{*}$, so that

$$
T^{*}(f d \omega)(y)=\int_{X} K(x, y) f(x) d \omega(x), \quad y \in X
$$


One of the immediate consequences of Theorem 1.1 (applied to both $T(f d \sigma)$ and $\left.T^{*}(f d \sigma)\right)$ is the following useful fact.

Corollary 1.1. Under the assumptions of Theorem 1.1, the functions $T(f d \sigma)(x)$, and $T^{*}(f d \sigma)(x)$ have equivalent $L_{\omega}^{q}$-norms, with the constants of equivalence independent of $f, \sigma$ and $\omega$. The same is true for weak $L_{\omega}^{q}$ norms when $1<q<\infty$.

In order to put our results about testing conditions in perspective, let us describe some of what is known about such conditions. For simplicity, we discuss this history primarily for strong-type estimates. The strong-type estimate (1.3) is of course equivalent to the dual estimate

$$
\left\|T^{*}(f d \omega)\right\|_{L_{\sigma}^{p^{\prime}}} \leq c\|f\|_{L_{\omega}^{q^{\prime}}}
$$

where $1 / p+1 / p^{\prime}=1$ and $1 / q+1 / q^{\prime}=1$. In [SWZ], it is shown that (1.3) holds for all $f$ if and only if both (1.3) and $\left(1.3^{*}\right)$ hold for all $f$ which are characteristic functions $\chi_{Q}$ of dyadic cubes, i.e., (1.3) holds if and only if both

$$
\begin{gathered}
\left(\int_{X} T\left(\chi_{Q} d \sigma\right)^{q} d \omega\right)^{1 / q} \leq c|Q|_{\sigma}^{1 / p}, \\
\left(\int_{X} T^{*}\left(\chi_{Q} d \omega\right)^{p^{\prime}} d \sigma\right)^{1 / p^{\prime}} \leq c|Q|_{\omega}^{1 / q^{\prime}}
\end{gathered}
$$

hold for all $Q \in \mathcal{D}$. This result is true without assuming any group structure for $X$. In case $X$ is Euclidean space with the usual metric, this sort of result was considered in [S3].

On the other hand, as is shown in [SWZ], it is not sufficient even in Euclidean space to replace the integration over $X$ in either (1.10) or (1.11) by integration over $Q$ (for dyadic $Q$ ). It is enough, in any homogeneous space, to replace the integration over $X$ by integration over appropriate enlargements of $Q$, for all dyadic $Q$; for specific results of this kind, see Theorem 1.2 of [SWZ]. Such results have useful applications to half-space estimates (e.g., Poisson integral estimates can be derived as corollaries of them, rather than requiring direct proofs; see the comments following Theorem 1.6 in [SWZ]).

It was recently shown in [WZ] that there is a " $B, B, B$ " characterization of (1.3) for balls $B$, i.e., that (1.3) holds if and only if both

$$
\begin{gathered}
\left(\int_{B} T\left(\chi_{B} d \sigma\right)^{q} d \omega\right)^{1 / q} \leq c|B|_{\sigma}^{1 / p}, \\
\left(\int_{B} T^{*}\left(\chi_{B} d \omega\right)^{p^{\prime}} d \sigma\right)^{1 / p^{\prime}} \leq c|B|_{\omega}^{1 / q^{\prime}}
\end{gathered}
$$

hold for all balls $B$ in $X$, without assuming any group structure for $X$. Previously, such a result was not known even in Euclidean space. As an application of Theorem 1.1, we will show that if $X$ has a group structure, then there is an analogous result with balls replaced by translates of dyadic cubes. More precisely, we will prove the following theorem.

Theorem 1.2. Let $1<p \leq q<\infty$, and let $\sigma$ and $\omega$ be locally finite Borel measures on a homogeneous space $X$ with a group operation "+" which satisfies (1.6). Let $T$ be defined by (1.1) with a kernel which satisfies (1.7). Then the strong-type 
estimate (1.3) holds for all Borel measurable functions $f$, with a constant $c$ that is independent of $f$, if and only if there exists a constant $c$ such that both

$$
\begin{gathered}
\left(\int_{Q+z} T\left(\chi_{Q+z} d \sigma\right)^{q} d \omega\right)^{1 / q} \leq c|Q+z|_{\sigma}^{1 / p} \\
\left(\int_{Q+z} T^{*}\left(\chi_{Q+z} d \omega\right)^{p^{\prime}} d \sigma\right)^{1 / p^{\prime}} \leq c|Q+z|_{\omega}^{1 / q^{\prime}}
\end{gathered}
$$

hold for all dyadic cubes $Q \in \mathcal{D}$ and all $z \in X$.

A similar result is proved in Theorem 1.3 of [SWZ], but with an additional condition on the kernel $K$ of $T$ (namely, condition (1.15) of [SWZ]).

For example, if $X$ is Euclidean space with the usual structure, and $\mathcal{D}$ is the usual grid of dyadic cubes, then $Q+z$ is also a cube whenever $Q \in \mathcal{D}$, and consequently, (1.3) is true if for all cubes $J$, dyadic or not, we assume the analogues of (1.14) and (1.15) with $Q+z$ replaced everywhere by $J$, for all cubes $J$. Moreover, since these analogues with $J$ are clearly necessary for (1.3), it follows that there is a " $J, J, J$ " characterization of (1.3) in Euclidean space, for all cubes $J$, dyadic or not. This answers a question raised in [S3, Remark 2].

We will also prove the following analogous result concerning the weak-type estimate (1.2).

Theorem 1.3. Let $1<p \leq q<\infty$, and let $\sigma$ and $\omega$ be locally finite Borel measures on a homogeneous space $X$ with a group operation "+" which satisfies (1.6). Let $T$ be defined by (1.1) with a kernel which satisfies (1.7). Then the weak-type estimate (1.2) holds for all Borel measurable functions $f$, with a constant $c$ that is independent of $f$, if and only if there exists a constant $c$ such that

$$
\left(\int_{Q+z} T^{*}\left(\chi_{Q+z} d \omega\right)^{p^{\prime}} d \sigma\right)^{1 / p^{\prime}} \leq c|Q+z|_{\omega}^{1 / q^{\prime}}
$$

for all dyadic cubes $Q \in \mathcal{D}$ and all $z \in X$.

Again, in Euclidean space with the usual structure, it then follows that the weaktype estimate (1.2) is true if and only if for all cubes $J$, dyadic or not, the analogue of (1.16) with $Q+z$ replaced everywhere by $J$ holds. In a slightly more restricted form, this sort of result was first proved in [S2]. In a homogeneous space, even without a group structure, a corresponding result of the $B, B, B$ type for balls $B$ was derived in [WZ], as mentioned earlier.

As the proof will show, Theorem 1.3 remains true for $p=1$ and $1<q<\infty$ if we replace (1.16) by

$$
\sigma \text { - } \operatorname{esssup}_{x \in Q+z} T^{*}\left(\chi_{Q+z} d \omega\right)(x) \leq C|Q+z|_{\omega}^{1 / q^{\prime}}
$$

for all $z \in X$ and $Q \in \mathcal{D}$.

Our pointwise results hold for kernels satisfying (1.7) in the (more difficult) case $p=q$.

Theorem 1.4. Let $1<p<\infty$, and let $\sigma$ and $\omega$ be locally finite Borel measures on a homogeneous space $X$ with a group operation "+" which satisfies (1.6). Let T 
be defined by (1.1) with a kernel which satisfies (1.7). Suppose $T(\mathbf{1} d \omega)(x) \in L_{\sigma, l o c}^{p^{\prime}}$ and

$$
T\left[T(\mathbf{1} d \omega)^{p^{\prime}} d \sigma\right](x) \leq C T(\mathbf{1} d \omega)(x) \quad d \sigma \text {-a.e. }
$$

Then the inequality

$$
\|T(f d \sigma)\|_{L_{\tilde{\omega}}^{p}} \leq c|| f \|_{L_{\sigma}^{p}}
$$

holds with a constant $c$ independent of $f$, where $\tilde{\omega}$ is defined by

$$
d \tilde{\omega}(x)=T(\mathbf{1} d \omega)^{p^{\prime}}(x) d \sigma(x)=\left[\int_{X} K(x, y) d \omega(y)\right]^{p^{\prime}} d \sigma(x) .
$$

Note that (1.19) always implies (1.3) with $p=q$ (see section 4, Corollary 4.2). There is also a version of Theorem 1.4 which is valid without the group structure assumption, with (1.18) replaced by its analogue involving the adjoint kernel (Lemma $4.2)$.

The proof of Theorem 1.4 makes use of our results on the discrete operator (Corollary 1.1) and is based on the useful inequality (see Lemma 4.1)

$$
[T(f d \sigma)(x)]^{p} \leq C T\left[f T(f d \sigma)^{p-1} d \sigma\right](x),
$$

where $f \in L_{\sigma, l o c}^{1}(f \geq 0), 1<p<\infty$, and $C$ depends only on $p, \kappa$, and the constant $C_{1}$ from (1.7). In the special case of Riesz potentials, Theorem 1.4 (with a more difficult proof based on weak-type inequalities and interpolation) was obtained in $[\mathrm{VW}]$.

We will show later by using Theorem 1.1 that Theorem 1.4 remains true if one replaces the pointwise condition (1.18) by its discrete analogue

$$
t_{\mathcal{G}}\left[t_{\mathcal{G}}(\mathbf{1} d \omega)^{p^{\prime}} d \sigma\right](x) \leq C t_{\mathcal{G}}(\mathbf{1} d \omega)(x) \quad d \sigma \text {-a.e. },
$$

where $C$ does not depend on the grid $\mathcal{G}=\mathcal{D}_{m}+z$.

Theorem 1.1 and some other facts about $t_{\mathcal{G}}$ are proved in section 2 . The proofs of Theorems 1.2 and 1.3 are given in section 3, the strong-type result being proved last as a corollary of the weak-type result and the $X, Q, Q$ fact indicated in (1.10) and (1.11). In both these sections, in order to save repetition, we will use some facts and arguments from [SW] and [SWZ], but we give explicit references when this is the case. Theorem 1.4 and some other related pointwise results are proved in section 4 .

\section{THE DISCRETE OPERATOR}

To prove Theorem 1.1, we will use some minor variants of $t_{\mathcal{G}}$ which we now describe. Let $\mathcal{G}$ denote the dyadic grid $\mathcal{D}_{m}+z$,

$$
\mathcal{G}=\left\{E_{j}^{k}+z: k \geq m, j \geq 1\right\}
$$

for fixed $m$ and $z$, where the cubes $E_{j}^{k}$ are as in (1.5). The value of $\lambda$ in (1.5) is assumed to be fixed: $\lambda=8 \kappa^{5}$. If $Q$ is a cube in $\mathcal{G}$, then $Q=E_{j}^{k}+z$ for some $j$ and $k$, and we will say that $Q$ is a cube of level $k$. Given $x \in X$ and a cube $Q$ in $\mathcal{G}$ which contains $x$, and given $M=1,2, \ldots$, define $P_{M}(Q, x)$ to be the cube in $\mathcal{G}$ which contains $x$ and which is $M$ levels below $Q$; if no such cube exists, i.e., if $Q$ 
belongs to one of the first $M-1$ levels of $\mathcal{G}$, define $P_{M}(Q, x)$ to be empty. Clearly, $P_{M}(Q, x) \subset Q$, due to the dyadic structure of $\mathcal{G}$. Let

$$
t_{\mathcal{G}, M}(f d \sigma)(x)=\sum_{Q \in \mathcal{G}: x \in Q} \phi_{Q} \int_{Q \backslash P_{M}(Q, x)} f(y) d \sigma(y),
$$

for $f \geq 0$, where $\phi_{Q}$ is defined to be $\phi\left(Q^{*}\right), Q^{*}$ being the containing ball of $Q$. Thus, if $x$ is fixed and $\left\{Q_{k}\right\}_{k \geq m}$ is the sequence of cubes of $\mathcal{G}$ containing $x, Q_{k}$ being of level $k$, then

$$
t_{\mathcal{G}, M}(f d \sigma)(x)=\sum_{k=m}^{m+M-1} \phi_{Q_{k}} \int_{Q_{k}} f d \sigma+\sum_{k=m+M}^{\infty} \phi_{Q_{k}} \int_{Q_{k} \backslash Q_{k-M}} f d \sigma .
$$

Note that when $M=1$, we have $t_{\mathcal{G}, 1}=t_{\mathcal{G}}$ and $P_{1}(Q, x)=P(Q, x)$. It is easy to see that

$$
t_{\mathcal{G}}(f d \sigma)(x)=\int_{X} k(x, y) f(y) d \sigma(y)
$$

where the kernel $k(x, y)$ is defined by $k(x, y)=\phi_{Q(x, y)}$; here $Q(x, y)$ is the smallest cube in $\mathcal{G}$ containing both $x$ and $y$. In particular, $k(x, y)$ is symmetric.

We also define, for $f \geq 0$,

$$
T_{\mathcal{G}}(f d \sigma)(x)=\sum_{Q \in \mathcal{G}: x \in Q} \phi_{Q} \int_{Q} f(y) d \sigma(y)
$$

and

$$
M_{\mathcal{G}}(f d \sigma)(x)=\sup _{Q \in \mathcal{G}: x \in Q} \phi_{Q} \int_{Q} f(y) d \sigma(y) .
$$

The operator $T_{\mathcal{G}}(f d \sigma)$ was defined in [SW] and [SWZ]. Its kernel $\widetilde{K}(x, y)$ can be written as

$$
\widetilde{K}(x, y)=\sum_{Q \in \mathcal{G}: Q \supset Q(x, y)} \phi_{Q}=\sum_{Q \in \mathcal{G}} \phi_{Q} \chi_{Q}(x) \chi_{Q}(y) .
$$

Lemma 2.1. For all $x$,

$$
\begin{aligned}
& c_{1} M_{\mathcal{G}}(f d \sigma)(x) \leq t_{\mathcal{G}}(f d \sigma)(x) \leq T_{\mathcal{G}}(f d \sigma)(x), \\
& t_{\mathcal{G}}(f d \sigma)(x) \leq t_{\mathcal{G}, M}(f d \sigma)(x) \leq c_{2} t_{\mathcal{G}}(f d \sigma)(x),
\end{aligned}
$$

where $c_{1}$ and $c_{2}$ are positive constants which depend only on $\kappa$ and the constants in (1.7), and $c_{2}$ also depends on $M$ (but $c_{1}, c_{2}$ do not depend on $\mathcal{G}, x, f$ or $\sigma$ ).

Proof. The second inequality in (i) is obvious. To prove the first inequality in (i), fix $x$ and a cube $Q$ in $\mathcal{G}$ which contains $x$. Write

$$
Q=Q_{m} \cup \bigcup_{k=m+1}^{l}\left(Q_{k} \backslash Q_{k-1}\right),
$$

where $Q_{k}$ is the cube of level $k$ containing $x$, and $Q_{l}=Q$. We will use the fact (see (4.3) of [SWZ]) that if $I$ and $J$ are cubes in $\mathcal{G}$ with $I \subset J$, then $\phi_{J} \leq C \phi_{I}$ with $C$ 
depending only on $\kappa$ and the constants in (1.7). Thus,

$$
\begin{gathered}
\phi_{Q} \int_{Q} f d \sigma=\phi_{Q}\left[\int_{Q_{m}} f d \sigma+\sum_{k=m+1}^{l} \int_{Q_{k} \backslash Q_{k-1}} f d \sigma\right] \\
\leq C\left[\phi_{Q_{m}} \int_{Q_{m}} f d \sigma+\sum_{k=m+1}^{l} \phi_{Q_{k}} \int_{Q_{k} \backslash Q_{k-1}} f d \sigma\right],
\end{gathered}
$$

(since $Q_{k} \subset Q$ and so $\phi_{Q} \leq C \phi_{Q_{k}}$ for all $k$ with $m \leq k \leq l$ )

$$
\leq C t_{\mathcal{G}}(f d \sigma)(x) \text { by }(2.1) \text { with } \quad M=1 .
$$

This completes the proof of part (i).

To prove part (ii), fix $x$ and let $\left\{Q_{k}\right\}_{k \geq m}$ be the cubes in $\mathcal{G}$ which contain $x$, with $Q_{k}$ a cube of level $k$. Fix $M$. The first estimate in (ii) is clearly true by using termby-term comparison of the sums (2.2) for $t_{\mathcal{G}, M}$ and $t_{\mathcal{G}}$, together with the nestedness property $Q_{k} \subset Q_{k+1}$. To prove the second estimate in part (ii), consider a term in the second sum on the right of (2.2), i.e., a value of $k$ with $k \geq m+M$. Then

$$
\begin{gathered}
\phi_{Q_{k}} \int_{Q_{k} \backslash Q_{k-M}} f d \sigma=\phi_{Q_{k}} \sum_{j=k-M+1}^{k} \int_{Q_{j} \backslash Q_{j-1}} f d \sigma \\
\leq C \sum_{j=k-M+1}^{k} \phi_{Q_{j}} \int_{Q_{j} \backslash Q_{j-1}} f d \sigma,
\end{gathered}
$$

as before since $Q_{j} \subset Q_{k}$ for $j \leq k$. Summing this over $k \geq m+M$ and changing the order of summation shows that the second sum in (2.2) is at most

$$
\begin{aligned}
& C \sum_{j=m+1}^{\infty}\left[\sum_{k=j}^{j+M-1} \mathbf{1}\right] \phi_{Q_{j}} \int_{Q_{j} \backslash Q_{j-1}} f d \sigma \\
= & C \sum_{j=m+1}^{\infty} M \phi_{Q_{j}} \int_{Q_{j} \backslash Q_{j-1}} f d \sigma \leq C M t_{\mathcal{G}}(f d \sigma)(x) .
\end{aligned}
$$

Also, for a term in the first sum in (2.2), i.e., for $k=m, \ldots, m+M-1$, as in (2.5),

$$
\begin{gathered}
\phi_{Q_{k}} \int_{Q_{k}} f d \sigma \leq C\left[\phi_{Q_{m}} \int_{Q_{m}} f d \sigma+\sum_{j=m+1}^{k} \phi_{Q_{j}} \int_{Q_{j} \backslash Q_{j-1}} f d \sigma\right] \\
\leq C t_{\mathcal{G}}(f d \sigma)(x) .
\end{gathered}
$$

Again adding over such $k$, we see that the first sum in (2.2) is also bounded by $C M t_{\mathcal{G}}(f d \sigma)(x)$. This proves the lemma.

Lemma 2.2. For all $x$,

$$
t_{\mathcal{G}}(f d \sigma)(x) \leq C\left\{\begin{array}{l}
T(f d \sigma)(x) \\
T^{*}(f d \sigma)(x)
\end{array}\right.
$$

with $C$ depending only on $\kappa$ and the constants in (1.7), but not on $\mathcal{G}, x, f$, or $\sigma$.

Note that the first estimate in Lemma 2.2 is the same as in the first part of Theorem 1.1. 
Proof. As mentioned earlier, similar estimates hold with $t_{\mathcal{G}}$ replaced by $T_{\mathcal{G}}$ provided we make an extra assumption on the kernel $K$. Fix $x$ and let $\left\{Q_{k}\right\}_{k \geq m}$ be the cubes in $\mathcal{G}$ which contain $x$, as usual, with $Q_{m-1}$ empty. Then

$$
t_{\mathcal{G}}(f d \sigma)(x)=\sum_{k \geq m} \phi_{Q_{k}} \int_{Q_{k} \backslash Q_{k-1}} f d \sigma .
$$

By (4.1) of [SWZ], $\phi_{Q_{k}} \leq C K(x, y)$ if $x, y \in Q_{k}$, with $C$ independent of $k, x, y$ and $\mathcal{G}$. Thus, the last sum is at most

$$
\begin{gathered}
C \sum_{k \geq m} \int_{Q_{k} \backslash Q_{k-1}} f(y) K(x, y) d \sigma(y) \\
\leq C \int_{X} f(y) K(x, y) d \sigma(y)=C T(f d \sigma)(x) .
\end{gathered}
$$

This proves the first inequality in the lemma. Since $K(x, y)$ may be replaced above by $K(y, x)$, the second inequality follows similarly, and Lemma 2.2 is proved.

For each $m \in \mathbf{Z}$, consider the truncated operator $T_{m}$ defined by

$$
T_{m}(f d \sigma)(x)=\int_{\left\{y \in X: d(x, y)>\lambda^{m}\right\}} K(x, y) f(y) d \sigma(y), \quad x \in X,
$$

as in $[\mathrm{SW}]$. We recall the notation

$$
\|f\|_{L_{\omega}^{q, \infty}}=\sup _{\rho>0} \rho|\{x \in X:|f(x)|>\rho\}|_{\omega}^{1 / q} .
$$

The following lemma, which improves Lemma 4.7 of [SW] and Lemma 3.1 of [SWZ], contains the remaining parts of Theorem 1.1.

Lemma 2.3. For $1 \leq q \leq \infty$,

$$
\left\|T_{m}(f d \sigma)\right\|_{L_{\omega}^{q}} \leq C \sup _{z \in X}\left\|t_{\mathcal{D}_{m}+z}(f d \sigma)\right\|_{L_{\omega}^{q}}
$$

and

$$
\|T(f d \sigma)\|_{L_{\omega}^{q}} \leq C \sup _{z \in X, m \in \mathbf{Z}}\left\|t_{\mathcal{D}_{m}+z}(f d \sigma)\right\|_{L_{\omega}^{q}}
$$

with $C$ independent of $m, f$ and $\omega$. For $1<q<\infty$, the same estimates hold with $\|\cdot\|_{L_{\omega}^{q}}$ replaced by $\|\cdot\|_{L_{\omega}^{q, \infty}}$.

Lemma 2.3 in fact holds for any norm. With $t_{\mathcal{D}_{m}+z}(f d \sigma)$ replaced by the larger function $T_{\mathcal{D}_{m}+z}(f d \sigma)$, Lemma 2.3 becomes the results in [SW] and [SWZ] mentioned above.

Proof. We adapt Lemma 4.7 of [SW], indicating the essential changes. Fix $m$, and for $k \in \mathbf{Z}, k>m$, define the truncation at infinity of the operator $T_{m}$ by

$$
T_{m}^{k}(f d \sigma)(x)=\int_{\left\{y \in X: \lambda^{m}<d(x, y) \leq \lambda^{k}\right\}} K(x, y) f(y) d \sigma(y) .
$$

Let $B_{k}$ denote the ball of radius $\lambda^{k}$ about the identity element in $X$. We claim that for $x \in B_{k}$,

$$
T_{m}^{k}(f d \sigma)(x) \leq C \frac{1}{\left|B_{k+3}\right|_{\mu}} \int_{B_{k+3}} t_{\mathcal{D}_{m}+z}(f d \sigma)(x) d \mu(z) .
$$


We recall that $t_{\mathcal{D}_{m}+z}(f d \sigma)(x)$ means $t_{\mathcal{D}_{m}+z, 1}(f d \sigma)(x)$, which by Lemma 2.1 (ii) is equivalent to $t_{\mathcal{D}_{m}+z, M}(f d \sigma)(x)$ uniformly in $m, z, x$ and $f$. Thus, for $x \in B_{k}, k>$ $m$, and $M$ to be chosen,

$$
\begin{gathered}
\frac{1}{\left|B_{k+3}\right|_{\mu}} \int_{B_{k+3}} t_{\mathcal{D}_{m}+z}(f d \sigma)(x) d \mu(z) \\
\geq C_{M} \frac{1}{\left|B_{k+3}\right|_{\mu}} \int_{B_{k+3}} t_{\mathcal{D}_{m}+z, M}(f d \sigma)(x) d \mu(z) \\
=C_{M} \int_{X}\left(\frac{1}{\left|B_{k+3}\right|_{\mu}} \int_{B_{k+3}}\left[\sum_{\substack{Q \in \mathcal{D}_{m}, x, y \in Q+z \\
y \notin P_{M}(Q+z, x)}} \phi_{Q+z}\right] d \mu(z)\right) f(y) d \sigma(y)
\end{gathered}
$$

by (2.1). We point out that the cube $P_{M}(Q+z, x)$ depends on the grid, which is $\mathcal{D}_{m}+z$ in the inner sum above.

Momentarily fix $y$ with $\lambda^{m}<d(x, y) \leq \lambda^{k}$ and choose $l \in \mathbf{Z}$ with $\lambda^{l-1}<$ $d(x, y) \leq \lambda^{l}, m<l \leq k$. Let $\widetilde{\Omega}$ be the set of all $z \in B_{k+3}$ so that there is a cube $Q \in \mathcal{D}_{m}$ with $s(Q)=\lambda^{l+1}, x, y \in Q+z$ and $y \notin P_{M}(Q+z, x)$. For such a cube $Q$, we have $\phi_{Q+z} \geq C K(x, y)$, and so the last expression exceeds

$$
C_{M} \frac{|\widetilde{\Omega}|_{\mu}}{\left|B_{k+3}\right|_{\mu}} T_{m}^{k}(f d \sigma)(x) .
$$

This will imply (2.7) provided we also prove that

$$
|\widetilde{\Omega}|_{\mu} \geq C\left|B_{k+3}\right|_{\mu} .
$$

Before proving (2.8), we note that the conclusion of the lemma follows from (2.7) since Minkowski's inequality then implies

$$
\begin{aligned}
\left\|T_{m}^{k}(f d \sigma)\right\|_{L_{\omega}^{q}\left(B_{k}\right)} & \leq C \frac{1}{\left|B_{k+3}\right|_{\mu}} \int_{B_{k+3}}\left\|t_{\mathcal{D}_{m}+z}(f d \sigma)\right\|_{L_{\omega}^{q}} d \mu(z) \\
\leq & C \sup _{z \in X}\left\|t_{\mathcal{D}_{m}+z}(f d \sigma)\right\|_{L_{\omega}^{q}}
\end{aligned}
$$

and the first inequality of the lemma follows by letting $k \rightarrow \infty$. The second inequality follows from the first by letting $m \rightarrow-\infty$. (Here and below we assume that $\sigma(\{x\})=0$ for all $x \in X$ when letting $m \rightarrow-\infty$ and using the monotone convergence theorem, because the domain of integration will be $X \backslash\{x\}$, which cannot be replaced by $X$ if $x$ is a joint atom of $\omega$ and $\sigma$.)

The analogous weak-type estimates are proved similarly since $\|\cdot\|_{L_{\omega}^{q, \infty}}$ is a norm for $1<q<\infty$.

It remains to prove (2.8). The proof is similar to that of (4.9) of [SW]. Let

$$
\Gamma=\left\{j: E_{j}^{l+1} \cap B\left(x, \lambda^{k+2}\right) \neq \emptyset\right\},
$$

where $\left\{E_{j}^{i}\right\}_{i \geq m, j \geq 1}$ are the cubes in $\mathcal{D}_{M}$. In particular,

$$
B\left(x_{j}^{l+1}, \lambda^{l+1}\right) \subset E_{j}^{l+1} \subset B\left(x_{j}^{l+1}, \lambda^{l+2}\right) .
$$

Now suppose that $z \in-B\left(x_{j}^{l+1}, \lambda^{l}\right)+x$. Then both $x, y \in E_{j}^{l+1}+z$ as in [SW, p. 868]. We claim that $y \notin P_{M}\left(E_{j}^{l+1}+z, x\right)$ if $M$ is sufficiently large and fixed, $M=4$ for example. We may assume that $l+1 \geq M+m$, since otherwise there 
is nothing to prove. Since $x-z \in E_{j}^{l+1}$, we then have $x-z \in E_{i}^{l+1-M}$ for some $i$, and therefore $P_{M}\left(E_{j}^{l+1}+z, x\right)=E_{i}^{l+1-M}+z$. If $y \in E_{i}^{l+1-M}+z$, then both $d\left(y-z, x_{i}^{l+1-M}\right) \leq \lambda^{l+2-M}$ and $d\left(x-z, x_{i}^{l+1-M}\right) \leq \lambda^{l+2-M}$, and consequently

$$
\begin{aligned}
\lambda^{l-1} \leq d(x, y) & =d(x-z, y-z) \leq \kappa\left[\lambda^{l+2-M}+\lambda^{l+2-M}\right] \\
& =2 \kappa \lambda^{l+2-M}<\lambda^{l+3-M}
\end{aligned}
$$

which is impossible if $M=4$. This proves our claim.

It follows that if $j \in \Gamma$ and $M=4$, then $-B\left(x_{j}^{l+1}, \lambda^{l}\right)+x$ satisfies all the requirements for being a subset of $\widetilde{\Omega}$ except possibly for being a subset of $B_{k+3}$. The argument to show that it is a subset of $B_{k+3}$ and the rest of the verification of (2.8) are the same as in [SW], and we will not repeat them here. This completes the proof of Lemma 2.3, and so also of Theorem 1.1.

\section{Proofs of the testing Results}

In order to prove (1.2) for $1<q<\infty$, it is enough by the weak-type statement in Lemma 2.3 to show that

$$
\left\|t_{\mathcal{G}}(f d \sigma)\right\|_{L_{\omega}^{q, \infty}} \leq C\|f\|_{L_{\sigma}^{p}}
$$

with $C$ independent of $\mathcal{G}$ and $f$. In case $p>1$, we shall use the following result.

Theorem 3.1. Suppose that $1<p \leq q<\infty, \omega$ and $\sigma$ are nonnegative measures on a homogeneous space $(X, d, \mu)$, and $t_{\mathcal{G}}(f d \sigma)$ is the dyadic operator defined by (2.1) with $M=1$. Then the weak-type inequality

$$
\left\|t_{\mathcal{G}}(f d \sigma)\right\|_{L_{\omega}^{q, \infty}} \leq c_{1}\|f\|_{L_{\sigma}^{p}}
$$

holds for all $f \geq 0$, with $c_{1}$ independent of $\mathcal{G}$ and $f$, if and only if

$$
\left(\int_{Q} t_{\mathcal{G}}\left(\chi_{Q} d \omega\right)(x)^{p^{\prime}} d \sigma(x)\right)^{1 / p^{\prime}} \leq c_{2}|Q|_{\omega}^{1 / q^{\prime}}
$$

for all $Q$ in $\mathcal{G}$ with $c_{2}$ independent of $Q$ and $\mathcal{G}$. The constants $c_{1}$ and $c_{2}$ are equivalent.

We do need to assume that $X$ has a group structure in Theorem 3.1. Moreover, there is an analogous result with $t_{\mathcal{G}}$ replaced everywhere by $T_{\mathcal{G}}$; its proof is slightly simpler than the one we shall give for $t_{\mathcal{G}}$.

Proof of Theorem 3.1. We first show that (3.3) implies (3.2). Fix $f \geq 0, \mathcal{G}$ and $p, q$ with $1<p \leq q<\infty$. We may assume without loss of generality that $\mathcal{G}$ is a (finite) collection consisting of a finite number of cubes at a certain level together with all dyadic subcubes of these. The first estimate of Lemma 1.1 (i) remains valid for such a collection. Since $\mathcal{G}$ is finite, $t_{\mathcal{G}}(f d \sigma)$ is bounded if $f \in L_{\sigma}^{p}$.

For $\rho>0$, let

$$
\Omega_{\rho}=\left\{x \in X: t_{\mathcal{G}}(f d \sigma)(x)>\rho\right\}
$$

Note that $\Omega_{\rho}$ is the union of cubes in $\mathcal{G}$, since if $x \in \Omega_{\rho}$ and $\left\{Q_{i}\right\}_{i \geq 1}$ is the sequence of cubes in $\mathcal{G}$ satisfying $x \in Q_{i}, Q_{i} \subset Q_{i+1}$ and $Q_{0}=\emptyset$, then 


$$
\begin{aligned}
\rho<t_{\mathcal{G}}(f d \sigma)(x) & =\sum_{i \geq 1} \phi_{Q_{i}} \int_{Q_{i} \backslash Q_{i-1}} f d \sigma \\
& =t_{\mathcal{G}}(f d \sigma)(y) \quad \text { if } \quad y \in Q_{1},
\end{aligned}
$$

and consequently, $Q_{1} \subset \Omega_{\rho}$.

Fix $\rho>0$ and a large positive number $L$ to be chosen. Write $\Omega_{\rho / L}=\bigcup_{j} Q_{j}$, where the $Q_{j}$ are the maximal cubes in $\mathcal{G}$ which are contained in $\Omega_{\rho / L}$. By maximality, the $Q_{j}$ are pairwise disjoint. We claim that

$$
t_{\mathcal{G}}\left(\chi_{Q_{j}^{c}} f d \sigma\right)(x) \leq \rho / 2 \quad \text { for all } \quad x \in Q_{j},
$$

if $L$ is large enough (independent of $\mathcal{G}, x, j$ and $f$ ). In case $Q_{j}$ happens to be one of the top level cubes in $\mathcal{G}$, we have $t_{\mathcal{G}}\left(\chi_{Q_{j}^{c}} f d \sigma\right)=0$ in $Q_{j}$, and so (3.4) is then obvious. If $Q_{j}$ is not a top level cube in $\mathcal{G}$, let $\widetilde{Q}_{j}$ be the next largest cube in $\mathcal{G}$ which contains $Q_{j}$. By maximality, there is a point $\xi \in \widetilde{Q}_{j} \cap \Omega_{\rho / L}^{c}$. If $x \in Q_{j}$, then by the dyadic structure,

$$
\begin{gathered}
t_{\mathcal{G}}\left(\chi_{Q_{j}^{c}} f d \sigma\right)(x)=\sum_{Q \in \mathcal{G}: Q \supset \widetilde{Q}_{j}} \phi_{Q} \int_{Q \backslash P(Q, x)} f d \sigma \\
=\phi_{\widetilde{Q}_{j}} \int_{\widetilde{Q}_{j} \backslash Q_{j}} f d \sigma+\sum_{Q \in \mathcal{G}: Q \supsetneqq \widetilde{Q}_{j}} \phi_{Q} \int_{Q \backslash P(Q, x)} f d \sigma=I+I I .
\end{gathered}
$$

We have

$$
I \leq \phi_{\widetilde{Q}_{j}} \int_{\widetilde{Q}_{j}} f d \sigma \leq M_{\mathcal{G}}(f d \sigma)(\xi) \leq C t_{\mathcal{G}}(f d \sigma)(\xi)
$$

since $\xi \in \widetilde{Q}_{j}$ and by Lemma 1.1 (ii), and so

$$
I \leq C \frac{\rho}{L}<\frac{\rho}{4} \quad \text { if } \quad L>4 C .
$$

Also, if $Q \supsetneqq \widetilde{Q}_{j}$ and $x \in Q_{j}$, then $P(Q, x)=P(Q, \xi)$, and therefore

$$
\begin{aligned}
I I \leq & \sum_{Q \in \mathcal{G}: Q \supsetneqq \widetilde{Q}_{j}} \phi_{Q} \int_{Q \backslash P(Q, \xi)} f d \sigma \quad \text { if } \quad x \in \widetilde{Q}_{j} \\
& \leq t_{\mathcal{G}}(f d \sigma)(\xi)<\frac{\rho}{L}<\frac{\rho}{4} \quad \text { if } \quad L>4 .
\end{aligned}
$$

Hence, for large $L, I+I I<\rho / 4+\rho / 4=\rho / 2$ if $x \in Q_{j}$, and (3.4) is proved in all cases.

Thus if $x \in \Omega_{\rho} \cap Q_{j}$, then

$$
t_{\mathcal{G}}\left(\chi_{Q_{j}} f d \sigma\right)(x)=t_{\mathcal{G}}(f d \sigma)(x)-t_{\mathcal{G}}\left(\chi_{Q_{j}^{c}} f d \sigma\right)(x)>\rho-\rho / 2=\rho / 2 .
$$

By integrating over $\Omega_{\rho} \cap Q_{j}$, we obtain

$$
\frac{\rho}{2}\left|\Omega_{\rho} \cap Q_{j}\right|_{\omega} \leq \int_{Q_{j}} t_{\mathcal{G}}\left(\chi_{Q_{j}} f d \sigma\right) d \omega
$$

Since $\Omega_{\rho} \subset \Omega_{\rho / L}$,

$$
\Omega_{\rho}=\Omega_{\rho} \cap \Omega_{\rho / L}=\bigcup_{j}\left(\Omega_{\rho} \cap Q_{j}\right)
$$


Thus,

$$
\begin{aligned}
\frac{\rho}{2}\left|\Omega_{\rho}\right|_{\omega} & \leq \sum_{j} \frac{\rho}{2}\left|\Omega_{\rho} \cap Q_{j}\right|_{\omega} \\
& \leq \sum_{j} \int_{Q_{j}} t_{\mathcal{G}}\left(\chi_{Q_{j}} f d \sigma\right) d \omega \quad \text { by }(3.5) .
\end{aligned}
$$

Let us now show that $t_{\mathcal{G}}$ is self-adjoint. First note that if $x, y \in Q$ then the statements $y \in Q \backslash P(Q, x)$ and $x \in Q \backslash P(Q, y)$ are equivalent. Hence, for any $f, g \geq 0$,

$$
\begin{gathered}
\int_{X} t_{\mathcal{G}}(f d \sigma)(x) g(x) d \omega(x)=\int_{X}\left[\sum_{\substack{Q \in \mathcal{G} \\
x \in Q}} \phi_{Q} \int_{Q \backslash P(Q, x)} f(y) d \sigma(y)\right] g(x) d \omega(x) \\
=\int_{X}\left[\sum_{\substack{Q \in \mathcal{G} \\
y \in Q}} \phi_{Q} \int_{Q} \chi_{Q \backslash P(Q, x)}(y) g(x) d \omega(x)\right] f(y) d \sigma(y) \\
=\int_{X}\left[\sum_{\substack{Q \in \mathcal{G} \\
y \in Q}} \phi_{Q} \int_{Q \backslash P(Q, y)} g(x) d \omega(x)\right] f(y) d \sigma(y) \\
=\int_{X} t_{\mathcal{G}}(g d \omega)(y) f(y) d \sigma(y),
\end{gathered}
$$

as desired.

In particular, by (3.6),

$$
\begin{gathered}
\frac{\rho}{2}\left|\Omega_{\rho}\right|_{\omega} \leq \sum_{j} \int_{Q_{j}} t_{\mathcal{G}}\left(\chi_{Q_{j}} d \omega\right) f d \sigma \\
\leq \sum_{j}\left(\int_{Q_{j}} t_{\mathcal{G}}\left(\chi_{Q_{j}} d \omega\right)^{p^{\prime}} d \sigma\right)^{1 / p^{\prime}}\left(\int_{Q_{j}} f^{p} d \sigma\right)^{1 / p} \text { by Hölder's inequality } \\
\leq \sum_{j} c_{2}\left|Q_{j}\right|_{\omega}^{1 / q^{\prime}}\left(\int_{Q_{j}} f^{p} d \sigma\right)^{1 / p} \text { by }(3.3) \\
\leq c_{2}\left(\sum_{j}\left|Q_{j}\right|_{\omega}^{p^{\prime} / q^{\prime}}\right)^{1 / p^{\prime}}\left(\sum_{j} \int_{Q_{j}} f^{p} d \sigma\right)^{1 / p} \text { by Hölder's inequality } \\
\leq c_{2}\left(\sum_{j}\left|Q_{j}\right|_{\omega}\right)^{1 / q^{\prime}}\left(\sum_{j} \int_{Q_{j}} f^{p} d \sigma\right)^{1 / p} \text { since } p^{\prime} \geq q^{\prime} \\
\leq c_{2}\left|\Omega_{\rho / L}\right|_{\omega}^{1 / q^{\prime}}\|f\|_{L_{\sigma}^{p}}
\end{gathered}
$$


since the $Q_{j}$ are pairwise disjoint and $\bigcup Q_{j}=\Omega_{\rho / L}$. Therefore,

$$
\rho^{q}\left|\Omega_{\rho}\right|_{\omega}=\rho^{q-1} \cdot \rho\left|\Omega_{\rho}\right|_{\omega} \leq 2 c_{2} L^{q-1}\left\{\left(\frac{\rho}{L}\right)^{q}\left|\Omega_{\rho / L}\right|_{\omega}\right\}^{1 / q^{\prime}}\|f\|_{L_{\sigma}^{p}}
$$

and then, for any $N>0$, since $0<\rho<N$ implies that $0<\rho / L<N$, we obtain

$$
\sup _{0<\rho<N} \rho^{q}\left|\Omega_{\rho}\right|_{\omega} \leq 2 c_{2} L^{q-1}\left\{\sup _{0<\rho<N} \rho^{q}\left|\Omega_{\rho}\right|_{\omega}\right\}^{1 / q^{\prime}}\|f\|_{L_{\sigma}^{p}} .
$$

Since $\Omega_{\rho}$ is a union of cubes in $\mathcal{G}$ and $\mathcal{G}$ is finite,

$$
\left|\Omega_{\rho}\right|_{\omega} \leq \sum_{Q \in \mathcal{G}}|Q|_{\omega}=A<\infty \text { for all } \rho>0
$$

and so

$$
\sup _{0<\rho<N} \rho^{q}\left|\Omega_{\rho}\right|_{\omega} \leq N^{q} A<\infty \text { for any } N<\infty .
$$

Thus, by dividing in (3.7), we obtain

$$
\left\{\sup _{0<\rho<N} \rho^{q}\left|\Omega_{\rho}\right|_{\omega}\right\}^{1 / q} \leq 2 c_{2} L^{q-1}\|f\|_{L_{\sigma}^{p}}
$$

and (3.2) follows with $c_{1}=2 c_{2} L^{q-1}$ by letting $N \rightarrow \infty$ and the number of cubes in $\mathcal{G}$ increase.

Conversely, to show that (3.2) implies (3.3), fix $Q$ and note that

$$
\begin{gathered}
\left(\int_{X} t_{\mathcal{G}}\left(\chi_{Q} d \omega\right)^{p^{\prime}} d \sigma\right)^{1 / p^{\prime}}=\sup _{\substack{f: f \geq 0 \\
\|f\|_{L_{\sigma}^{p}=1}^{p}}} \int_{X} t_{\mathcal{G}}\left(\chi_{Q} d \omega\right) f d \sigma \\
=\sup _{\substack{f f: f_{0} \geq 0 \\
\|f\|_{L_{\sigma}^{p}=1}}} \int_{Q} t_{\mathcal{G}}(f d \sigma) d \omega \text { since } t_{\mathcal{G}} \text { is self-adjoint } \\
=\sup _{\substack{f: f \geq 0 \\
\|f\|_{L_{\sigma}^{p}=1}}} \int_{0}^{\infty}\left|\left\{x \in Q: t_{\mathcal{G}}(f d \sigma)(x)>\rho\right\}\right|_{\omega} d \rho \\
\leq \sup _{\substack{f: f \geq 0 \\
\|f\|_{L_{\sigma}^{p}=1}}} \int_{0}^{\infty} \min \left\{|Q|_{\omega},\left(c_{1}\|f\|_{L_{\sigma}^{p}} / \rho\right)^{q}\right\} d \rho \text { by }(3.2) \\
\leq \int_{0}^{c_{1}|Q|_{\omega}^{-1 / q}}|Q|_{\omega} d \rho+\int_{c_{1}|Q|_{\omega}^{-1 / q}}^{\infty}\left(\frac{c_{1}}{\rho}\right)^{q} d \rho \\
=2 c_{1}|Q|_{\omega}^{1 / q^{\prime}} .
\end{gathered}
$$

This proves (3.3) with $c_{2}=2 c_{1}$, and so completes the proof of Theorem 3.1.

Remark. Minor modifications of the proof show that Theorem 3.1 remains true for $p=1$ and $1<q<\infty$ if (3.3) is replaced by

$$
\sigma \text { - } \operatorname{ess}_{x \in Q} \sup _{\mathcal{G}}\left(\chi_{Q} d \omega\right)(x) \leq c_{2}|Q|_{\omega}^{1 / q^{\prime}}
$$


Proof of Theorem 1.3. As noted before, in order to prove (1.2), it is enough to prove (3.1) (or (3.2)) with a constant independent of $\mathcal{G}$ and $f$, where $\mathcal{G}=\mathcal{D}_{m}+z$. Hypothesis (1.16) together with the second part of Lemma 2.2 implies that

$$
\left(\int_{Q} t_{\mathcal{G}}\left(\chi_{Q} d \omega\right)(x)^{p^{\prime}} d \sigma(x)\right)^{1 / p^{\prime}} \leq C|Q|_{\omega}^{1 / q^{\prime}}
$$

for all $Q \in \mathcal{G}$ for any such $\mathcal{G}$, with $C$ independent of $Q$ and $\mathcal{G}$. This is the same as (3.3), and consequently, the desired result follows immediately from Theorem 3.1. Conversely, the fact that (1.2) implies (1.16) can be proved by making minor modifications in the corresponding argument used for Theorem 3.1. We omit the details.

Proof of Theorem 1.2. Testing (1.3) and $\left(1.3^{*}\right)$ with $f=\chi_{Q+z}$ gives (1.14) and (1.15) immediately. It is then enough to prove that (1.14) and (1.15) together imply (1.3), and so, as explained in the introduction, it is enough to show that they imply both (1.10) and (1.11) for every $Q \in \mathcal{D}$. To check (1.11), fix $Q \in \mathcal{D}$ and apply the same argument that we used above in the proof of Theorem 3.1 to show that (3.2) implies (3.3), but with $t_{\mathcal{G}}$ replaced by $T^{*}$. Since the adjoint of $T^{*}$ is $T$, and since the weak-type estimate (1.2) follows by Theorem 1.3 from (1.15) (note that (1.15) and (1.16) are the same), we obtain (1.11) in this way. Similarly, by applying Theorem (1.3) to $T^{*}$ and using (1.14), we see that (1.10) holds, and the proof is complete.

\section{Pointwise Results}

To prove Theorem 1.4 we will need several lemmas. As in sections 1-3, we assume that $K$ satisfies (1.7) and that all annuli in $X$ are nonempty. From the latter assumption it follows (see $[\mathrm{SW}]$ ) that the constant $C_{2}$ in (1.7) may be chosen to be arbitrarily large. We will assume that $C_{2}>2 \kappa$, where $\kappa$ is the quasi-metric constant in (1.4).

Lemma 4.1. Suppose $1<p<\infty$ and $\omega$ is a locally finite Borel measure on $X$. Then

$$
\left[\int_{X} K(x, y) d \omega(y)\right]^{p} \leq C \int_{X} K(x, y)\left[\int_{X} K(y, z) d \omega(z)\right]^{p-1} d \omega(y)
$$

where $C$ depends only on $\kappa, C_{1}$, and $p$.

Proof. We will first prove (4.1) for $x \in X$ such that $\int_{X} K(x, y) d \omega(y)<\infty$. Let

$$
I=\int_{X} K(x, y) d \omega(y)
$$

and

$$
J=\int_{X} K(x, y)\left[\int_{X} K(y, z) d \omega(z)\right]^{p-1} d \omega(y) .
$$

Let us show that $I^{p} \leq C J$, where $C$ depends only on $\kappa, C_{1}$, and $p$. In what follows we may assume $J \leq I^{p}$. (Otherwise the desired inequality is obvious.)

First we consider the case $1<p \leq 2$. Then

$$
I^{p}=\int_{X} K(x, y)\left[\int_{X} K(x, z) d \omega(z)\right]^{p-1} d \omega(y) \leq I_{1}+I_{2},
$$


where

$$
I_{1}=\int_{X} K(x, y)\left[\int_{\left\{z: d(y, z) \leq C_{2} d(x, z)\right\}} K(x, z) d \omega(z)\right]^{p-1} d \omega(y)
$$

and

$$
I_{2}=\int_{X} K(x, y)\left[\int_{\left\{z: d(y, z)>C_{2} d(x, z)\right\}} K(x, z) d \omega(z)\right]^{p-1} d \omega(y) .
$$

By (1.7), in the inside integral for $I_{1}$ we have $K(x, z) \leq C_{1} K(y, z)$. Thus,

$$
I_{1} \leq \int_{X} K(x, y)\left[\int_{X} C_{1} K(y, z) d \omega(z)\right]^{p-1} d \omega(y)=C_{1}^{p-1} J .
$$

To estimate $I_{2}$, we use Hölder's inequality with exponents $1 /(p-1)$ and $1 /(2-p)$ :

$$
I_{2} \leq I^{2-p}\left\{\int_{X} K(x, y)\left[\int_{\left\{z: d(y, z)>C_{2} d(x, z)\right\}} K(x, z) d \omega(z)\right] d \omega(y)\right\}^{p-1} .
$$

By Fubini's theorem

$$
\begin{aligned}
& \int_{X} K(x, y)\left[\int_{\left\{z: d(y, z)>C_{2} d(x, z)\right\}} K(x, z) d \omega(z)\right] d \omega(y) \\
= & \int_{X} K(x, z)\left[\int_{\left\{y: d(y, z)>C_{2} d(x, z)\right\}} K(x, y) d \omega(y)\right] d \omega(z) .
\end{aligned}
$$

Obviously,

$$
\int_{\left\{y: d(y, z)>C_{2} d(x, z)\right\}} K(x, y) d \omega(y) \leq I .
$$

Since $p-1 \leq 1$, we obtain from this

$$
\int_{\left\{y: d(y, z)>C_{2} d(x, z)\right\}} K(x, y) d \omega(y) \leq\left[\int_{\left\{y: d(y, z)>C_{2} d(x, z)\right\}} K(x, y) d \omega(y)\right]^{p-1} I^{2-p} .
$$

We next note that

$$
\left\{y: d(y, z)>C_{2} d(x, z)\right\} \subset\left\{y: d(y, z) \leq C_{2} d(x, y)\right\} .
$$

Otherwise, if $d(y, z)>C_{2} d(x, z)$ and $d(y, z)>C_{2} d(x, y)$, then by (1.4)

$$
2 d(y, z)>C_{2} d(x, z)+C_{2} d(x, y) \geq \frac{C_{2}}{\kappa} d(y, z),
$$

which contradicts the fact that $C_{2}>2 \kappa$. Thus

$$
\begin{gathered}
\int_{\left\{y: d(y, z)>C_{2} d(x, z)\right\}} K(x, y) d \omega(y) \leq \int_{\left\{y: d(z, y) \leq C_{2} d(x, y)\right\}} K(x, y) d \omega(y) \\
\leq C_{1} \int_{X} K(z, y) d \omega(y)
\end{gathered}
$$


by (1.7). From the preceding inequalities, we have

$$
\begin{aligned}
& \int_{X} K(x, y)\left[\int_{\left\{z: d(y, z)>C_{2} d(x, z)\right\}} K(x, z) d \omega(z)\right] d \omega(y) \\
& \leq I^{2-p} \int_{X} K(x, z)\left[C_{1} \int_{X} K(z, y) d \omega(z)\right]^{p-1} d \omega(z) .
\end{aligned}
$$

From this and (4.3) it follows that

$$
\begin{gathered}
I_{2} \leq I^{2-p}\left\{I^{2-p} \int_{X} K(x, z)\left[C_{1} \int_{X} K(z, y) d \omega(y)\right]^{p-1} d \omega(z)\right\}^{p-1} \\
=C_{1}^{(p-1)^{2}} I^{p(2-p)} J^{p-1} .
\end{gathered}
$$

Combining the estimates for $I_{1}$ and $I_{2}$, we obtain

$$
I^{p} \leq I_{1}+I_{2} \leq C_{1}^{p-1} J+C_{1}^{(p-1)^{2}} I^{p(2-p)} J^{p-1},
$$

where $1<p \leq 2$. We have assumed $J \leq I^{p}<\infty$, which implies $J \leq I^{p(2-p)} J^{p-1}$. From this and the preceding inequality we have

$$
I^{p} \leq\left(C_{1}^{p-1}+C_{1}^{(p-1)^{2}}\right) I^{p(2-p)} J^{p-1}
$$

which yields

$$
I^{p} \leq C_{1}^{p-1}\left(1+C_{1}^{(p-1)(2-p)}\right)^{1 /(p-1)} J
$$

for $1<p \leq 2$.

We now prove that $I^{p} \leq C J$ in the easier case $p>2$. We assume again that $I<\infty$. Using a similar inequality for $p=2$ proved above and Hölder's inequality with exponent $p-1$, we have

$$
\begin{gathered}
I^{2} \leq C \int_{X} K(x, y)\left[\int_{X} K(y, z) d \omega(z)\right] d \omega(y) \\
\leq C\left\{\int_{X} K(x, y)\left[\int_{X} K(y, z) d \omega(z)\right]^{p-1} d \omega(y)\right\}^{1 /(p-1)} \\
\times\left\{\int_{X} K(x, y) d \omega(y)\right\}^{(p-2) /(p-1)}=C J^{1 /(p-1)} I^{(p-2) /(p-1)},
\end{gathered}
$$

which clearly implies $I^{p} \leq C J$.

It remains to prove (4.1) for $x$ such that $I=\int_{X} K(x, y) d \omega(y)=\infty$. We define the truncated kernel $K_{N}(N=1,2, \ldots)$ by $K_{N}(x, y)=\min [K(x, y), N]$. It is easily seen that $K_{N}$ satisfies (1.7) with the same constants $C_{1}$ and $C_{2}$ independent of $N$. Since $\omega$ is locally finite, we see that for all balls $B$ in $X$

$$
\int_{B} K_{N}(x, y) d \omega(y)<\infty, \quad \text { and } \sup _{B, N} \int_{B} K_{N}(x, y) d \omega(y)=\infty .
$$

Applying the argument used above, we obtain

$$
\left[\int_{B} K_{N}(x, y) d \omega(y)\right]^{p} \leq C \int_{B} K_{N}(x, y)\left[\int_{B} K_{N}(y, z) d \omega(z)\right]^{p-1} d \omega(y),
$$


with a constant $C$ independent of $B$ and $N$. Taking the supremum over all $B$ on both sides of the preceding inequality and letting $N \rightarrow \infty$, we see that the righthand side of (4.1) is also infinite. Thus (4.1) holds for all $x \in X$. The proof of Lemma 4.1 is complete.

Applying Lemma 4.1 to $d \omega(x)=f(x) d \sigma(x)$, we immediately obtain the following statement.

Corollary 4.1. Suppose $1<p<\infty$, and $f \in L_{\sigma, l o c}^{1}(f \geq 0)$. Suppose $K$ satisfies (1.7). Then

$$
[T(f d \sigma)(x)]^{p} \leq C T\left[f T(f d \sigma)^{p-1} d \sigma\right](x),
$$

where $C$ depends only on $\kappa, C_{1}$, and $p$.

Let

$$
d \omega_{1}(y)=\left[T^{*}(\mathbf{1} d \omega)\right]^{p^{\prime}}(y) d \sigma(y)=\left[\int_{X} K(x, y) d \omega(x)\right]^{p^{\prime}} d \sigma(y)
$$

Corollary 4.2. Suppose that the kernel $K$ satisfies (1.7). Suppose $1<p<\infty$ and $f \in L_{\sigma}^{p}$. Then the inequality

$$
\|T(f d \sigma)\|_{L_{\omega}^{p}} \leq c|| f \|_{L_{\sigma}^{p}}
$$

holds with a constant $c$ independent of $f$ if the similar inequality

$$
\|T(f d \sigma)\|_{L_{\omega_{1}}^{p}} \leq c_{1}\|f\|_{L_{\sigma}^{p}}
$$

holds with $\omega_{1}$ in place of $\omega$ and a constant $c_{1}$ independent of $f$. Moreover,

$$
\|T(f d \sigma)\|_{L_{\omega}^{p}} \leq C\|T(f d \sigma)\|_{L_{\omega_{1}}^{p}}^{1 / p^{\prime}}\|f\|_{L_{\sigma}^{p}}^{1 / p}
$$

where $C$ is independent of $f, \sigma$, and $\omega$.

Proof. By Corollary 4.1,

$$
[T(f d \sigma)(x)]^{p} \leq C T\left[f T(f d \sigma)^{p-1} d \sigma\right](x) .
$$

From this by Fubini's theorem and Hölder's inequality it follows that

$$
\begin{gathered}
\int_{X} T(f d \sigma)^{p} d \omega \leq C \int_{X} T\left[f T(f d \sigma)^{p-1} d \sigma\right] d \omega \\
=C \int_{X} f T(f d \sigma)^{p-1} T^{*}(\mathbf{1} d \omega) d \sigma \leq C\|f\|_{L_{\sigma}^{p}}\|T(f d \sigma)\|_{L_{\omega_{1}}^{p}}^{p-1},
\end{gathered}
$$

which proves (4.8). Then obviously (4.7) and (4.8) yield (4.6). The proof of Corollary 4.2 is complete.

The next lemma is an analogue of Theorem 1.4 with (1.18) replaced by

$$
T^{*}\left[T^{*}(\mathbf{1} d \omega)^{p^{\prime}} d \sigma\right](x) \leq C T^{*}(\mathbf{1} d \omega)(x) \quad d \sigma \text {-a.e. }
$$

Here

$$
T^{*}(f d \omega)(y)=\int_{X} K(x, y) f(x) d \omega(x), \quad y \in X .
$$

We set $\omega_{0}=\omega$, and

$$
d \omega_{j}(y)=T^{*}\left(\mathbf{1} d \omega_{j-1}\right)^{p^{\prime}}(y) d \sigma(y)=\left[\int_{X} K(x, y) d \omega_{j-1}(x)\right]^{p^{\prime}} d \sigma(y)
$$

for $j=1,2$. 
Lemma 4.2. Suppose $T^{*}(\mathbf{1} d \omega) \in L_{\sigma, l o c}^{p^{\prime}}$ and (4.10) holds, i.e.,

$$
T^{*}\left(\mathbf{1} d \omega_{1}\right)(x) \leq C T^{*}(\mathbf{1} d \omega)(x) \quad d \sigma \text {-a.e. }
$$

Then

$$
\|T(f d \sigma)\|_{L_{\omega_{j}}^{p}} \leq c_{j}\|f\|_{L_{\sigma}^{p}}
$$

where $c_{j}$ is independent of $f(j=0,1,2)$.

Proof. It suffices to prove (4.12) for $j=1$, since the case $j=0$ then follows by Corollary 4.2 and the case $j=2$ by (4.11). Note that our hypothesis that $T^{*}(\mathbf{1} d \omega) \in L_{\sigma, l o c}^{p^{\prime}}$ implies that $\omega_{1}$ is locally finite, and (4.11) implies that $\omega_{2} \leq C \omega_{1}$ as measures. We denote by $T_{N}$ the integral operator

$$
T_{N}(f d \sigma)(x)=\int_{X} K_{N}(x, y) f(y) d \sigma(y)
$$

with the truncated kernel $K_{N}(x, y)=\min [K(x, y), N]$ in place of $K(x, y)$. As was mentioned in the proof of Lemma 4.1, $K_{N}$ satisfies (1.7) with the same constants $C_{1}$ and $C_{2}$ independent of $N$. We will first prove the inequality

$$
\left\|T_{N}(f d \sigma)\right\|_{L_{\omega_{1}}^{p}} \leq c\|f\|_{L_{\sigma}^{p}},
$$

with a constant $c$ independent of $f \in L_{\sigma}^{p}$ and $N$. Then (4.12) will follow by letting $N \rightarrow \infty$.

We can assume without loss of generality that $f \geq 0$ vanishes outside some fixed ball $B$ in $X$. By Corollary 4.1 with $T_{N}$ in place of $T$, we have

$$
\int_{X} T_{N}(f d \sigma)^{p} d \omega_{1} \leq C \int_{X} T_{N}\left[f T_{N}(f d \sigma)^{p-1}\right] d \omega_{1}
$$

Using Fubini's theorem and Hölder's inequality, as in the proof of Corollary 4.2, we get

$$
\begin{aligned}
& \int_{X} T_{N}(f d \sigma)^{p} d \omega_{1} \leq C \int_{B} f T_{N}(f d \sigma)^{p-1} T_{N}^{*}\left(\mathbf{1} d \omega_{1}\right) d \sigma \\
& \leq C\|f\|_{L_{\sigma}^{p}}\left(\int_{B} T_{N}(f d \sigma)^{p} d \omega_{2}\right)^{1 / p^{\prime}} .
\end{aligned}
$$

By (4.11),

$$
\int_{B} T_{N}(f d \sigma)^{p} d \omega_{2} \leq C \int_{B} T_{N}(f d \sigma)^{p} d \omega_{1}<\infty ;
$$

the right-hand side of the preceding inequality is finite because $T_{N}(f d \sigma)$ is obviously bounded and $\omega_{1}$ is assumed to be locally finite. Hence the left-hand side of (4.14) is also finite, and it follows from (4.14) and the first inequality in (4.15) that

$$
\int_{X} T_{N}(f d \sigma)^{p} d \omega_{1} \leq C\|f\|_{L_{\sigma}^{p}}\left(\int_{X} T_{N}(f d \sigma)^{p} d \omega_{1}\right)^{1 / p^{\prime}}<\infty .
$$

The preceding inequality implies (4.13), which completes the proof of Lemma 4.2.

By applying Lemma 4.2 with the roles of $T$ and $T^{*}$ interchanged (including the $T^{*}$ which appears in the definition of $\omega_{1}$ ), and then applying Corollary 1.1 to the resulting conclusion, we complete the proof of Theorem 1.4. 
Taking into account that $t_{\mathcal{G}}$ is self-adjoint, we now show that we can replace $T^{*}$ in the pointwise condition (4.10) of Lemma 4.2 above by its discrete version involving $t_{\mathcal{G}}$, where $\mathcal{G}=\mathcal{D}_{m}+z$.

Corollary 4.3. Suppose $t_{\mathcal{G}}(\mathbf{1} d \omega) \in L_{\sigma, l o c}^{p^{\prime}}$ and

$$
t_{\mathcal{G}}\left[t_{\mathcal{G}}(\mathbf{1} d \omega)^{p^{\prime}} d \sigma\right](x) \leq C t_{\mathcal{G}}(\mathbf{1} d \omega)(x) \quad d \sigma \text {-a.e. },
$$

where $C$ does not depend on the grid $\mathcal{G}=\mathcal{D}_{m}+z$. Then (1.19) holds.

Proof. Corollary 4.3 follows from Theorem 1.1 and Theorem 1.4 (applied to $t_{\mathcal{G}}$ ) provided we know that the kernel $k(x, y)$ of $t_{\mathcal{G}}$ satisfies (1.7). To show that this is the case, we note that, as was mentioned in section $2, k(x, y)=\phi_{Q(x, y)}$, where $Q(x, y)$ is the smallest cube in $\mathcal{G}$ containing both $x$ and $y$. Given $x, y$ and $x, y^{\prime}$ with $d\left(x, y^{\prime}\right) \leq C d(x, y)$, it is easily seen that the smallest dyadic cube $Q^{\prime}=Q\left(x, y^{\prime}\right)$ containing both $x$ and $y^{\prime}$ is a subset of a fixed enlargement of $Q=Q(x, y)$. The same is obviously true for the corresponding containing balls $B^{\prime}$ and $B$ of $Q^{\prime}$ and $Q$. Since $k\left(x, y^{\prime}\right)=\phi_{Q^{\prime}}=\phi\left(B^{\prime}\right)$ and $k(x, y)=\phi_{Q}=\phi(B)$ by the definition of $\phi_{Q}$, the desired inequality $k(x, y) \leq C k\left(x^{\prime}, y\right)$ then follows from the following properties of $\phi(B)$ : If $B$ and $B^{\prime}$ are balls and $B^{\prime} \subset B$, then $\phi(B) \leq C \phi\left(B^{\prime}\right)$; also, for any ball $B$ we have that $\phi(B) \leq C \phi(2 B)$ (see [SWZ]). Thus $\phi(B) \leq C \phi\left(B^{\prime}\right)$, which proves the first property in (1.7). The second one reduces to the first one because $k(x, y)$ is symmetric. This completes the proof of Corollary 4.3 .

\section{REFERENCES}

[C] M. Christ, A $T(b)$ theorem with remarks on analytic capacity and the Cauchy integral, Colloq. Math. 60, 61 (1990), 601-628. MR 92k:42020

[CW] R. Coifman and G. Weiss, Extensions of Hardy spaces and their use in analysis, Bull. Amer. Math. Soc. 83 (1977), 569-645. MR 56:6264

$[\mathrm{KV}]$ N. J. Kalton and I. E. Verbitsky, Nonlinear equations and weighted norm inequalities, Trans. Amer. Math. Soc. (to appear).

[MV] V. G. Maz'ya and I. E. Verbitsky, Capacitary inequalities for fractional integrals, with applications to partial differential equations and Sobolev multipliers, Arkiv för Matem. 33 (1995), 81- 115. MR 96i:26021

[S1] E. T. Sawyer, A characterization of a two-weight norm inequality for maximal operators, Studia Math. 75 (1982), 1-11. MR 84i:42032

[S2] E. T. Sawyer, A two weight weak type inequality for fractional integrals, Trans. Amer. Math. Soc. 281 (1984), 339-345. MR 85j:26010

[S3] E. T. Sawyer, A characterization of two weight norm inequalities for fractional and Poisson integrals, Trans. Amer. Math. 308 (1988), 533-545. MR 89d:26009

[SW] E. T. Sawyer and R. L. Wheeden, Weighted inequalities for fractional integrals on Euclidean and homogeneous spaces, Amer. J. Math. 114 (1992), 813-874. MR 94i:42024

[SWZ] E. T. Sawyer, R. L. Wheeden, and S. Zhao, Weighted norm inequalities for operators of potential type and fractional maximal functions, Potential Analysis 5 (1996), 523-580. CMP 97:09

[VW] I. E. Verbitsky and R. L. Wheeden, Weighted trace inequalities for fractional integrals and applications to semilinear equations, J. Funct. Analysis 129 (1995), 221-241. MR 95m:42025

[WZ] R. L. Wheeden and S. Zhao, Weak-type estimates for operators of potential type, Studia Math. 119 (1996), 149-160. MR 97d:42013

Department of Mathematics, University of Missouri, Columbia, Missouri 65211

E-mail address: igor@math.missouri.edu

Department of Mathematics, Rutgers University, New Brunswick, New Jersey 08903

E-mail address: wheeden@math.rutgers.edu 